\title{
Statistical Thermodynamics
}

\author{
Anatol Malijevský \\ Department of Physical Chemistry, Institute of Chemical Technology, Prague \\ Czech Republic
}

\section{Introduction}

This chapter deals with the statistical thermodynamics (statistical mechanics) a modern alternative of the classical (phenomenological) thermodynamics. Its aim is to determine thermodynamic properties of matter from forces acting among molecules. Roots of the discipline are in kinetic theory of gases and are connected with the names Maxwelland Boltzmann. Father of the statistical thermodynamics is Gibbs who introduced its concepts such as the statistical ensemble and others, that have been used up to present.

Nothing can express an importance of the statistical thermodynamics better than the words of Richard Feynman Feynman et al. (2006), the Nobel Prize winner in physics: If, in some cataclysm, all of scientific knowledge were to be destroyed, and only one sentence passed on to the next generations of creatures, what statement would contain the most information in the fewest words? I believe it is the atomic hypothesis (or the atomic fact, or whatever you wish to call it) that All things are made of atoms - little particles that move around in perpetual motion, attracting each other when they are a little distance apart, but repelling upon being squeezed into one another.

In that one sentence, you will see, there is an enormous amount of information about the world, if just a little imagination and thinking are applied.

The chapter is organized as follows. Next section contains axioms of the phenomenological thermodynamics. Basic concepts and axioms of the statistical thermodynamics and relations between the partition function and thermodynamic quantities are in Section 3. Section 4 deals with the ideal gas and Section 5 with the ideal crystal. Intermolecular forces are discussed in Section 6. Section 7 is devoted to the virial expansion and Section 8 to the theories of dense gases and liquids. The final section comments axioms of phenomenological thermodynamics in the light of the statistical thermodynamics.

\section{Principles of phenomenological thermodynamics}

The phenomenological thermodynamics or simply thermodynamics is a discipline that deals with the thermodynamic system, a macroscopic part of the world. The thermodynamic state of system is given by a limited number of thermodynamic variables. In the simplest case of one-component, one-phase system it is for example volume of the system, amount of substance (e.g. in moles) and temperature. Thermodynamics studies changes of thermodynamic quantities such as pressure, internal energy, entropy, e.t.c. with thermodynamic variables. 
The phenomenological thermodynamics is based on six axioms (or postulates if you wish to call them), four of them are called the laws of thermodynamics:

- Axiom of existence of the thermodynamic equilibrium

For thermodynamic system at unchained external conditions there exists a state of the thermodynamic equilibrium in which its macroscopic parameters remain constant in time. The thermodynamic system at unchained external conditions always reaches the state of the thermodynamic equilibrium.

- Axiom of additivity

Energy of the thermodynamic system is a sum of energies of its macroscopic parts. This axiom allows to define extensive and intensive thermodynamic quantities.

- The zeroth law of thermodynamics

When two systems are in the thermal equilibrium, i.e. no heat flows from one system to the other during their thermal contact, then both systems have the same temperature as an intensive thermodynamic parameter. If system $A$ has the same temperature as system $B$ and system $B$ has the same temperature as system $C$, then system A also has the same temperature as system $C$ (temperature is transitive).

- The first law of thermodynamics

There is a function of state called internal energy $U$. For its total differential $\mathrm{d} U$ we write

$$
\mathrm{d} U=\mathrm{d} W+\mathrm{d} Q,
$$

where the symbols $₫ Q$ and $₫ W$ are not total differentials but represent infinitesimal values of heat $Q$ and work $W$ supplied to the system.

- The second law of thermodynamics

There is a function of state called entropy $S$. For its total differential $\mathrm{d} S$ we write

$$
\begin{array}{ll}
\mathrm{d} S=\frac{\mathrm{d} Q}{\mathrm{~T}}, & \text { [reversible process }] \\
\mathrm{d} S>\frac{\mathrm{d} Q}{\mathrm{~T}}, & \text { [irreversible process]. }
\end{array}
$$

\section{- The third law of thermodynamics}

At temperature of $0 \mathrm{~K}$, entropy of a pure substance in its most stable crystalline form is zero

$$
\lim _{T \rightarrow 0} S=0 .
$$

This postulate supplements the second law of thermodynamics by defining a natural referential value of entropy. The third law of thermodynamics implies that temperature of $0 \mathrm{~K}$ cannot be attained by any process with a finite number of steps.

Phenomenological thermodynamics using its axioms radically reduces an amount of experimental effort necessary for a determination of the values of thermodynamic quantities. For example enthalpy or entropy of a pure fluid need not be measured at each temperature and pressure but they can be calculated from an equation of state and a temperature dependence of the isobaric heat capacity of ideal gas. However, empirical constants in an equation of state and in the heat capacity must be obtained experimentally. 


\section{Principles of statistical thermodynamics}

\subsection{Basic concepts}

The statistical thermodynamics considers thermodynamic system as an assembly of a very large number (of the order of $10^{23}$ ) of mutually interacting particles (usually molecules). It uses the following concepts:

- Microscopic state of system

The microscopic state of thermodynamic system is given by positions and velocities of all particles in the language of the Newton mechanics, or by the quantum states of the system in the language of quantum mechanics. There is a huge number of microscopic states that correspond to a given thermodynamic (macroscopic) state of the system.

- Statistical ensemble

Statistical ensemble is a collection of all systems that are in the same thermodynamic state but in the different microscopic states.

- Microcanonical ensemble or NVE ensemble is a collection of all systems at a given number of particles $N$, volume $V$ and energy $E$.

- Canonical ensemble or NVT ensemble is a collection of all systems at a given number of particles $N$, volume $V$ and temperature $T$.

There is a number of ensembles, e.g. the grandcanonical $(\mu \mathbf{V T})$ or isothermal isobaric (NPT) that will not be considered in this work.

- Time average of thermodynamic quantity

The time average $\bar{X}_{\tau}$ of a thermodynamic quantity $X$ is given by

$$
\bar{X}_{\tau}=\frac{1}{\tau} \int_{0}^{\tau} X(t) \mathrm{d} t
$$

where $X(t)$ is a value of $X$ at time $t$ and, $\tau$ is a time interval of a measurement.

- Ensemble average of thermodynamic quantity

The ensemble average $\bar{X}_{s}$ of a thermodynamic quantity $X$ is given by

$$
\bar{X}_{s}=\sum_{i} P_{i} X_{i}
$$

where $X_{i}$ is a value in the quantum state $i$, and $P_{i}$ is the probability of the quantum state.

\subsection{Axioms of the statistical thermodynamics}

The statistical thermodynamics is bases on two axioms:

Axiom on equivalence of average values

It is postulated that the time average of thermodynamic quantity $X$ is equivalent to its ensemble average

$$
\bar{X}_{\tau}=\bar{X}_{s}
$$

Axiom on probability

Probability $P_{i}$ of a quantum state $i$ is only a function of energy of the quantum state, $E_{i}$,

$$
P_{i}=f\left(E_{i}\right) .
$$




\subsection{Probability in the microcanonical and canonical ensemble}

From Eq.(8) relations between the probability and energy can be derived:

Probability in the microcanonical ensemble

All the microscopic states in the microcanonical ensemble have the same energy. Therefore,

$$
P_{i}=\frac{1}{W} \quad \text { for } \quad i=1,2, \ldots, W,
$$

where $W$ is a number of microscopical states (the statistical weight) of the microcanonical ensemble.

Probability in the canonical ensemble

In the canonical ensemble it holds

$$
P_{i}=\frac{\exp \left(-\beta E_{i}\right)}{Q}
$$

where $\beta=\frac{1}{k_{B} T}, k_{B}$ is the Boltzmann constant, $T$ temperature and $Q$ is the partition function

$$
Q=\sum_{i} \exp \left(-\beta E_{i}\right)
$$

where the sum is over the microscopic states of the canonical ensemble.

\subsection{The partition function and thermodynamic quantities}

If the partition function is known thermodynamic quantities may be determined. The following relations between the partition function in the canonical ensemble and thermodynamic quantities can be derived

$$
\begin{aligned}
A & =-k_{B} T \ln Q \\
U & =k_{B} T^{2}\left(\frac{\partial \ln Q}{\partial T}\right)_{V} \\
S & =k_{B} \ln Q+k_{B} T\left(\frac{\partial \ln Q}{\partial T}\right)_{V} . \\
C_{V} & =\left(\frac{\partial U}{\partial T}\right)_{V}=k_{B} T^{2} \frac{\partial^{2} \ln Q}{\partial T^{2}}+2 k_{B} T\left(\frac{\partial \ln Q}{\partial T}\right)_{V}, \\
p & =-\left(\frac{\partial A}{\partial V}\right)_{T}=k_{B} T\left(\frac{\partial \ln Q}{\partial V}\right)_{T}, \\
H & =U+p V=k_{B} T^{2}\left(\frac{\partial \ln Q}{\partial T}\right)_{V}+V k_{B} T\left(\frac{\partial \ln Q}{\partial V}\right)_{T}, \\
G & =A+p V=-k_{B} T \ln Q+V k_{B} T\left(\frac{\partial \ln Q}{\partial V}\right)_{T}^{\prime} \\
C_{p} & =\left(\frac{\partial H}{\partial T}\right)_{V}=C_{V}+V k_{B} \frac{\partial^{2} \ln Q}{\partial V \partial T} .
\end{aligned}
$$

$A$ is Helmholtz free energy, $U$ internal energy, $S$ entropy, $C_{V}$ isochoric heat capacity, $p$ pressure, $H$ enthalpy, $G$ Gibbs free energy and $C_{p}$ isobaric heat capacity. 
Unfortunately, the partition function is known only for the simplest cases such as the ideal gas (Section 4) or the ideal crystal (Section 5). In all the other cases, real gases and liquids considered here, it can be determined only approximatively.

\subsection{Probability and entropy}

A relation between entropy $S$ and probabilities $P_{i}$ of quantum states of a system can be proved in the canonical ensemble

$$
S=-k_{B} \sum_{i} P_{i} \ln P_{i} .
$$

For the microcanonical ensemble a similar relation holds

$$
S=k_{B} \ln W,
$$

where $W$ is a number of accessible states. This equation (with log instead of $\ln$ ) is written in the grave of Ludwig Boltzmann in Central Cemetery in Vienna, Austria.

\section{Ideal gas}

The ideal gas is in statistical thermodynamics modelled by a assembly of particles that do not mutually interact. Then the energy of $\mathrm{i}$-th quantum state of system, $E_{i}$, is a sum of energies of individual particles

$$
E_{i}=\sum_{i=1}^{N} \epsilon_{i, j} .
$$

In this way a problem of a determination of the partition function of system is dramatically simplified. For one-component system of $N$ molecules it holds

$$
Q=\frac{q^{N}}{N !}
$$

where

$$
q=\sum_{j} \exp \left(-\beta \epsilon_{j}\right)
$$

is the partition function of molecule.

The partition function of molecule may be further simplified. The energy of molecule can be approximated by a sum of the translational $\epsilon_{\text {trans }}$, the rotational $\epsilon_{\text {rot }}$, the vibrational $\epsilon_{\mathrm{vib}}$, and the electronic $\epsilon_{\mathrm{el}}$ contributions (subscript $j$ in $\epsilon_{j}$ is omitted for simplicity of notation)

$$
\epsilon=\epsilon_{0}+\epsilon_{\text {trans }}+\epsilon_{\text {rot }}+\epsilon_{\mathrm{vib}}+\epsilon_{e l},
$$

where $\epsilon_{0}$ is the zero point energy. The partition function of system then becomes a product

$$
Q=\frac{\exp \left(-N \beta \epsilon_{0}\right)}{N !} q_{\text {trans }} q_{\mathrm{rot}} q_{\mathrm{vib}} q_{\mathrm{el}}
$$

Consequently all thermodynamic quantities of the ideal gas become sums of the corresponding contributions. For example the Helmholtz free energy is

$$
\begin{aligned}
A & =-k_{B} T \ln Q \\
& =k_{B} T \ln N !+U_{0}-N k_{B} T \ln q_{\mathrm{tr}}-N k_{B} T \ln q_{\mathrm{rot}}-N k_{B} T \ln q_{\mathrm{vib}}-N k_{B} T \ln q_{\mathrm{el}} \\
& =k_{B} T \ln N !+U_{0}+A_{\mathrm{tr}}+A_{\mathrm{rot}}+A_{\mathrm{vib}}+A_{\mathrm{el}},
\end{aligned}
$$

where $U_{0}=N \epsilon_{0}$ and $A_{\mathrm{tr}}, A_{\mathrm{rot}}, A_{\mathrm{vib}}, A_{\mathrm{el}}$ are the translational, rotational, vibrational, electronic contributions to the Helmholtz free energy, respectively. 


\subsection{Translational contributions}

Translational motions of a molecule are modelled by a particle in a box. For its energy a solution of the Schrödinger equation gives

$$
\epsilon_{\mathrm{tr}}=\frac{h^{2}}{8 m}\left(\frac{n_{x}^{2}}{a^{2}}+\frac{n_{y}^{2}}{b^{2}}+\frac{n_{z}^{2}}{c^{2}}\right),
$$

where $h$ is the Planck constant, $m$ mass of molecule, and $a b c=V$ where $V$ is volume of system. Quantities $n_{x}, n_{y}, n_{z}$ are the quantum numbers of translation. The partition function of translation is

$$
q_{\mathrm{tr}}=\left(\frac{2 \pi m k_{B} T}{h^{2}}\right)^{3 / 2} V .
$$

Translational contribution to the Helmholtz energy is

$$
A_{\mathrm{tr}}=-R T \ln q_{\mathrm{tr}}=-R T \ln \left(\lambda^{-3} V\right)
$$

where $R=N k_{B}$ is the gas constant and $\lambda=h / \sqrt{2 \pi m k_{B} T}$ is the Broglie wavelength.

The remaining thermodynamic functions are as follows

$$
\begin{aligned}
& S_{\mathrm{tr}}=-\left(\frac{\partial A_{\mathrm{tr}}}{\partial T}\right)_{V}=R \ln \left(\lambda^{-3} V\right)+\frac{3}{2} R, \\
& p_{\mathrm{tr}}=-\left(\frac{\partial A_{\mathrm{tr}}}{\partial V}\right)_{T}=\frac{R T}{V}, \\
& U_{\mathrm{tr}}=A_{\mathrm{tr}}+T S_{\mathrm{tr}}=\frac{3}{2} R T, \\
& H_{\mathrm{tr}}=U_{\mathrm{tr}}+p_{\mathrm{tr}} V=\frac{5}{2} R T, \\
& G_{\mathrm{tr}}=A_{\mathrm{tr}}+p_{\mathrm{tr}} V=-R T \ln \left(\lambda^{-3} V\right)+R T, \\
& C_{V, \mathrm{tr}}=\left(\frac{\partial U_{\mathrm{tr}}}{\partial T}\right)_{V}=\frac{3}{2} R, \\
& C_{p, \operatorname{tr}}=\left(\frac{\partial H_{\mathrm{tr}}}{\partial T}\right)_{p}=\frac{5}{2} R .
\end{aligned}
$$

\subsection{Rotational contributions}

Rotations of molecule are modelled by the rigid rotator. For linear molecules there are two independent axes of rotation, for non-linear molecules there are three.

\subsubsection{Linear molecules}

For the partiton function of rotation it holds

$$
q_{\mathrm{rot}}=\frac{8 \pi I k_{B} T}{\sigma h^{2}}
$$

where $\sigma$ is the symmetry number of molecule and $I$ its moment of inertia

$$
I=\sum_{1}^{n} m_{i} r_{i}^{2}
$$


with $n$ a number of atoms in molecule, $m_{i}$ their atomic masses and $r_{i}$ their distances from the center of mass. Contributions to the thermodynamic quantities are

$$
\begin{aligned}
A_{\text {rot }} & =-R T \ln q_{\text {rot }}=-R T \ln \left(\frac{8 \pi^{2} I k_{B} T}{\sigma h^{2}}\right), \\
S_{\text {rot }} & =R \ln \left(\frac{8 \pi^{2} I k_{B} T}{\sigma h^{2}}\right)+R, \\
p_{\text {rot }} & =0 \\
U_{\text {rot }} & =R T, \\
H_{\text {rot }} & =U_{\text {rot }} \\
G_{\text {rot }} & =F_{\text {rot }} \\
C_{V, \text { rot }} & =R, \\
C_{p, \text { rot }} & =C_{V, \text { rot }} .
\end{aligned}
$$

\subsubsection{Non-linear molecules}

The partition function of rotation of a non-linear molecule is

$$
q_{\mathrm{rot}}=\frac{1}{\sigma}\left(\frac{8 \pi^{2} k_{B} T}{h^{2}}\right)^{3 / 2}\left(\pi I_{A} I_{B} I_{C}\right)^{1 / 2},
$$

where $I_{A}, I_{B}$ and $I_{C}$ the principal moments of inertia. Contributions to the thermodynamic quantities are

$$
\begin{aligned}
A_{\text {rot }} & =-R T \ln q_{\text {rot }}=-R T \ln \left[\frac{1}{\sigma}\left(\frac{8 \pi^{2} k_{B} T}{h^{2}}\right)^{3 / 2}\left(\pi I_{A} I_{B} I_{C}\right)^{1 / 2}\right], \\
S_{\text {rot }} & =R \ln \left[\frac{1}{\sigma}\left(\frac{8 \pi^{2} k_{B} T}{h^{2}}\right)^{3 / 2}\left(\pi I_{A} I_{B} I_{C}\right)^{1 / 2}\right]+\frac{3}{2} R, \\
p_{\text {rot }} & =0 \\
U_{\text {rot }} & =\frac{3}{2} R T \\
H_{\text {rot }} & =U_{\text {rot }} \\
G_{\text {rot }} & =A_{\text {rot }} \\
C_{V, \text { rot }} & =\frac{3}{2} R, \\
C_{p, \text { rot }} & =C_{V, \text { rot }} .
\end{aligned}
$$

\subsection{Vibrational contributions}

Vibrations of atoms in molecule around their equilibrium states may be at not very high temperatures approximated by harmonic oscillators.

\subsubsection{Diatomic molecules}

In a diatomic molecule there is only one vibrational motion. Its partition function is

$$
q_{\mathrm{vib}}=\left[1-\exp \left(h v_{0} / k_{B} T\right)\right]^{-1},
$$


where $v_{0}$ is the fundamental harmonic frequency. Vibrational contributions to thermodynamic quantities are

$$
\begin{aligned}
A_{\mathrm{vib}} & =-R T \ln q_{\mathrm{vib}}=R T \ln \left(1-e^{-x}\right), \\
S_{\mathrm{vib}} & =R \frac{x e^{-x}}{1-e^{-x}}-R \ln \left(1-e^{-x}\right), \\
p_{\mathrm{vib}} & =0, \\
U_{\mathrm{vib}} & =R T \frac{x e^{-x}}{1-e^{-x}} \\
H_{\mathrm{vib}} & =U_{\mathrm{vib}} \\
G_{\mathrm{vib}} & =A_{\mathrm{vib}} \\
C_{V, \mathrm{vib}} & =R \frac{x^{2} e^{-x}}{\left(1-e^{-x}\right)^{2}}, \\
C_{p, \mathrm{vib}} & =C_{V, \mathrm{vib}},
\end{aligned}
$$

where $x=\frac{h v_{0}}{k_{B} T}$.

\subsubsection{Polyatomic molecules}

In $n$-atomic molecule there is $f$ fundamental harmonic frequencies $v_{i}$ where

$$
f= \begin{cases}3 n-5 & \text { linear molecule } \\ 3 n-6 & \text { non-linear molecule }\end{cases}
$$

The partition function of vibration is

$$
q_{\mathrm{vib}}=\prod_{i=1}^{f} \frac{1}{1-\exp \left(-h v_{i} / k_{B} T\right)} .
$$

For the thermodynamic functions of vibration we get

$$
\begin{aligned}
A_{\mathrm{vib}} & =-R T \ln q_{\mathrm{vib}}=R T \sum_{i=1}^{f} \ln \left(1-e^{-x_{i}}\right), \\
S_{\mathrm{vib}} & =R \sum_{i=1}^{f} \frac{x_{i} e^{-x_{i}}}{1-e^{-x_{i}}}-R \sum_{i=1}^{f} \ln \left(1-e^{-x_{i}}\right), \\
p_{\mathrm{vib}} & =0 \\
U_{\mathrm{vib}} & =R T \sum_{i=1}^{f} \frac{x_{i} e^{-x_{i}}}{1-e^{-x_{i}}} \\
H_{\mathrm{vib}} & =U_{\mathrm{vib}}, \\
G_{\mathrm{vib}} & =A_{\mathrm{vib}}, \\
C_{V, \mathrm{vib}} & =R \sum_{i=1}^{f} \frac{x_{i}^{2} e^{-x_{i}}}{\left(1-e^{-x_{i}}\right)^{2}} \\
C_{p, \mathrm{vib}} & =C_{V, \mathrm{vib}},
\end{aligned}
$$


where $x_{i}=\frac{h v_{i}}{k_{B} T}$.

\subsection{Electronic contributions}

The electronic partition function reads

$$
q_{\mathrm{el}}=\sum_{\ell=0}^{\infty} g_{\mathrm{el}, \ell} e^{-\varepsilon_{\mathrm{el}, \ell} / k_{B} T},
$$

where $\varepsilon_{\mathrm{el}, \ell}$ the energy level $\ell$, and $g_{\mathrm{el}, \ell}$ is its degeneracy. In most cases the electronic contributions to the thermodynamic functions are negligible at not very hight temperatures. Therefore they are not written here.

\subsection{Ideal gas mixture}

Let us consider two-component mixture of $N_{1}$ non-interacting molecules of component 1 and $\mathrm{N}_{2}$ non-interacting molecules of component 2 (extension to the case of a multi-component mixture is straightforward). The partition function of mixture is

$$
Q=\frac{q_{1}^{N_{1}}}{N_{1} !} \frac{q_{2}^{N_{2}}}{N_{2} !}
$$

where $q_{1}$ and $q_{2}$ are the partition functions of molecules 1 and 2, respectively. Let us denote $X_{m, i}$ the molar thermodynamic quantity of pure component $i, i=1,2$ and $x_{i}=\frac{N_{i}}{N_{1}+N_{2}}$ its mole fraction. Then

$$
\begin{aligned}
A & =R T\left(x_{1} \ln x_{1}+x_{2} \ln x_{2}\right)+x_{1} A_{m, 1}+x_{2} A_{m, 2} \\
S & =-R\left(x_{1} \ln x_{1}+x_{2} \ln x_{2}\right)+x_{1} S_{m, 1}+x_{2} S_{m, 2}, \\
G & =R T\left(x_{1} \ln x_{1}+x_{2} \ln x_{2}\right)+x_{1} G_{m, 1}+x_{2} G_{m, 2}, \\
p & =x_{1} p_{m, 1}+x_{2} p_{m, 2}=x_{1} \frac{R T}{V_{m}}+x_{2} \frac{R T}{V_{m}}, \\
U & =x_{1} U_{m, 1}+x_{2} U_{m, 2}, \\
H & =x_{1} H_{m, 1}+x_{2} H_{m, 2}, \\
C_{V} & =x_{1} C_{V m, 1}+x_{2} C_{V m, 2}, \\
C_{p} & =x_{1} C_{p m, 1}+x_{2} C_{p m, 2} .
\end{aligned}
$$

\section{Ideal crystal}

We will call the ideal crystal an assembly of molecules displayed in a regular lattice without any impurities or lattice deformations. Distances among lattice centers will not depend on temperature and pressure. For simplicity we will consider one-atomic molecules. The partition function of crystal is

$$
Q=e^{-U_{0} / k_{B} T} Q_{\mathrm{vib}}
$$

where $U_{0}$ is the lattice energy.

We will discuss here two models of the ideal crystal: the Einstein approximation and the Debye approximation. 


\subsection{Einstein model}

An older and simpler Einstein model is based on the following postulates

1. Vibrations of molecules are independent:

$$
Q_{\mathrm{vib}}=q_{\mathrm{vib}}^{N}
$$

where $q_{\text {vib }}$ is the vibrational partition function of molecule.

2. Vibrations are isotropic:

$$
q_{\mathrm{vib}}=q_{x} q_{y} q_{z}=q_{x}^{3}
$$

3. Vibrations are harmonical

$$
q_{x}=\sum_{v=0}^{\infty} e^{-\epsilon_{v} / k_{B} T}
$$

where

$$
\epsilon_{v}=h v\left(v+\frac{1}{2}\right)
$$

is the energy in quantum state $v$ and $v$ is the fundamental vibrational frequency.

Combining these equations one obtains

$$
Q=e^{-U_{0} / k_{B} T}\left(\frac{e^{-\Theta_{E} /(2 T)}}{1-e^{-\Theta_{E} / T}}\right)^{3 N}
$$

where

$$
\Theta_{E}=\frac{h v}{k_{B}}
$$

is the Einstein characteristic temperature.

For the isochoric heat capacity it follows

$$
C_{V}=3 N k_{B}\left(\frac{\Theta_{E}}{T}\right)^{2} \frac{e^{-\Theta_{E} / T}}{\left(1-e^{-\Theta_{E} / T}\right)^{2}} .
$$

\subsection{Debye model}

Debye considers crystal as a huge molecule (i.e he replaces the postulates of independence and isotropy in the Einstein model) of an ideal gas; the postulate of harmonicity of vibrations remains. From these assumptions it can be derived for the partition function

$$
\ln Q=-\frac{U_{0}}{k_{B} T}-\frac{9}{8} N \frac{\Theta_{D}}{T}-9 N\left(\frac{T}{\Theta_{D}}\right)^{3} \int_{0}^{\Theta_{D} / T} x^{2} \ln \left(1-e^{-x}\right) \mathrm{d} x,
$$

where

$$
\Theta_{D}=\frac{h v_{\max }}{k_{B}}
$$

is the Debye characteristic temperature with $v_{\max }$ being the highest frequency of crystal.

For the isochoric heat capacity it follows

$$
C_{V}=3 R\left(4 D(u)-\frac{3 u}{e^{u}-1}\right) .
$$


where $u=\Theta_{D} / T$ and

$$
D(u)=\frac{3}{u^{3}} \int_{0}^{u} \frac{x^{3}}{e^{x}-1} \mathrm{~d} x .
$$

It can be proved that at low temperatures the heat capacity becomes a cubic function of temperature

$$
C_{V}=36 R\left(\frac{T}{\Theta_{D}}\right)^{3} \int_{0}^{\infty} \frac{x^{3}}{e^{x}-1} \mathrm{~d} x=a T^{3},
$$

while the Einstein model incorrectly gives

$$
C_{V}=3 R\left(\frac{\Theta_{E}}{T}\right)^{2} e^{-\Theta_{E} / T}
$$

Both models give a correct high-temperature limit (the Dulong-Petit law)

$$
C_{V}=3 R \text {. }
$$

The same is true for the zero temperature limit

$$
\lim _{T \rightarrow 0} C_{V}=0 .
$$

\subsection{Beyond the Debye model}

Both the Einstein and the Debye models assume harmonicity of lattice vibrations. This is not true at high temperatures near the melting point. The harmonic vibrations are not assumed in the lattice theories (the cell theory, the hole theory, ...) that used to be popular in forties and fifties of the last century for liquids. It was shown later that they are poor theories of liquids but very good theories for solids.

Thermodynamic functions cannot be obtained analytically in the lattice theories.

\section{Intermolecular forces}

Up to now forces acting among molecules have been ignored. In the ideal gas (Section 4) molecules are assumed to exert no forces upon each other. In the ideal crystal (Section 5) molecules are imprisoned in the lattice, and the intermolecular forces are counted indirectly in the lattice energy and in the Einstein or Debye temperature. For real gases and liquids the intermolecular force must be included explicitly.

\subsection{The configurational integral and the molecular interaction energy}

The partition function of the real gas or liquid may be written in a form

$$
Q=\frac{1}{N !} \exp \left(-N \beta \epsilon_{0}\right) q_{\text {int }}^{N}\left(\frac{2 \pi m k_{B} T}{h^{2}}\right)^{\frac{3}{2} N} Z .
$$

where $q_{\text {int }}=q_{\text {rot }} q_{\mathrm{vib}} q_{\mathrm{el}}$ is the partition function of the internal motions in molecule. Quantity $\mathrm{Z}$ is the configurational integral

$$
Z=\int_{(V)} \int_{(V)} \cdots \int_{(V)} \exp \left[-\beta u_{N}\left(\vec{r}_{1}, \vec{r}_{2}, \ldots, \vec{r}_{N}\right)\right] \mathrm{d} \vec{r}_{1} \mathrm{~d} \vec{r}_{2} \ldots \mathrm{d} \vec{r}_{N},
$$


where symbol

$$
\int_{(V)} \cdots \mathrm{d} \vec{r}_{i}=\int_{0}^{L} \int_{0}^{L} \int_{0}^{L} \cdots \mathrm{d} x_{i} \mathrm{~d} y_{i} \mathrm{~d} z_{i} \quad \text { and } \quad L^{3}=V .
$$

The quantity $u_{N}\left(\vec{r}_{1}, \vec{r}_{2}, \ldots, \vec{r}_{N}\right)$ is the potential energy of an assembly of $N$ molecules. Here and in Eq.(93) one-atomic molecules are assumed for simplicity. More generally, the potential energy is a function not only positions of centers of molecules $\vec{r}_{i}$ but also of their orientations $\vec{\omega}_{i}$. However, we will use the above simplified notation.

The interaction potential energy $u_{N}$ of system may be written as an expansion in two-body, three-body, e.t.c contributions

$$
\left.u_{N}\left(\vec{r}_{1}, \vec{r}_{2}, \ldots, \vec{r}_{N}\right)=\sum_{i<j} u_{2}\left(\vec{r}_{i}, \vec{r}_{j}\right)+\sum_{i<j<k} u_{3}\left(\vec{r}_{i}, \vec{r}_{j}, \vec{r}_{k}\right)\right)+\cdots
$$

Most often only the first term is considered. This approximation is called the rule of pairwise additivity

$$
u_{N}=\sum_{i<j} u_{2}\left(\vec{r}_{i}, \vec{r}_{j}\right),
$$

where $u_{2}$ is the pair intermolecular potential. The three-body potential $u_{3}$ is used rarely at very accurate calculations, and $u_{4}$ and higher order contributions are omitted as a rule.

\subsection{The pair intermolecular potential}

The pair potential depends of a distance between centers of two molecules $r$ and on their mutual orientation $\vec{\omega}$. For simplicity we will omit the angular dependence of the pair potential (it is true for the spherically symmetric molecules) in further text, and write

$$
u_{2}\left(\vec{r}_{i}, \vec{r}_{j}\right)=u_{2}\left(r_{i j}, \vec{\omega}_{i j}\right)=u(r)
$$

where subscripts 2 and $i j$ are omitted, too.

The following model pair potentials are most often used.

\subsubsection{Hard spheres}

It is after the ideal gas the simplest model. It ignores attractive interaction between molecules, and approximates strong repulsive interactions at low intermolecular distances by an infinite barrier

$$
u(r)=\left\{\begin{array}{cc}
\infty & r<\sigma \\
0 & r>\sigma
\end{array}\right.
$$

where $\sigma$ is a diameter of molecule.

\subsubsection{Square well potential}

Molecules behave like hard spheres surrounded by an area of attraction

$$
u(r)=\left\{\begin{array}{crr}
\infty & r<\sigma \\
-\epsilon & \sigma<r & <\lambda \sigma \\
0 & & r>\lambda \sigma
\end{array}\right.
$$

Here $\sigma$ is a hard-sphere diameter, $\epsilon$ a depth of the attractive well, and the attraction region ranges from $\sigma$ to $\lambda \sigma$. 


\subsubsection{Lennard-Jones potential}

This well known pair intermolecular potential realistically describes a dependence of pair potential energy on distance

$$
u(r)=4 \epsilon\left[\left(\frac{\sigma}{r}\right)^{12}-\left(\frac{\sigma}{r}\right)^{6}\right] .
$$

$\epsilon$ is a depth of potential at minimum, and $2^{1 / 6} \sigma$ is its position.

More generally, the Lennard-Jones $n-m$ potential is

$$
u(r)=4 \epsilon\left[\left(\frac{\sigma}{r}\right)^{n}-\left(\frac{\sigma}{r}\right)^{m}\right] .
$$

\subsubsection{Pair potentials of non-spherical molecules}

There are analogues of hard spheres for non-spherical particles: hard diatomics or dumbbells made of two fused hard spheres, hard triatomics, hard multiatomics, hard spherocylinders, hard ellipsoids, and so on.

Examples of soft pair potentials are Lennard-Jones multiatomics, molecules whose atoms interact according to the Lennard-Jones potential (98).

Another example is the Stockmayer potential, the Lennard-Jones potential with an indebted dipole moment

$$
u\left(r, \theta_{1}, \theta_{2}, \phi\right)=4 \epsilon\left[\left(\frac{\sigma}{r}\right)^{12}-\left(\frac{\sigma}{r}\right)^{6}\right]-\frac{\mu^{2}}{r^{3}}\left[2 \cos \theta_{1} \cos \theta_{2}-\sin \theta_{1} \sin \theta_{2} \cos \phi\right],
$$

where $\mu$ is the dipole moment.

\subsubsection{Pair potentials of real molecules}

The above model pair potentials, especially the Lennard-Jones potential and its extensions, may be used to calculate properties of the real substances. In this case their parameters, for example $\epsilon$ and $\sigma$, are fitted to the experimental data such as the second virial coefficients, rare-gas transport properties and molecular properties.

More sophisticated approach involving a realistic dependence on the interparticle separation with a number of adjustable parameters was used by Aziz, see Aziz (1984) and references therein.

For simple molecules, there is a fully theoretical approach without any adjustable parameters utilizing the first principle quantum mechanics calculations, see for example Slaviček et al. (2003) and references therein.

\subsection{The three-body potential}

The three-body intermolecular interactions are caused by polarizablilities of molecules. The simplest and the most often used is the Axilrod-Teller-Muto term

$$
u(r, s, t)=\frac{v}{r s t}\left(3 \cos \theta_{1} \cos \theta_{2} \cos \theta_{3}+1\right),
$$

where $v$ is a strength parameter. It is a first term (DDD, dipole-dipole-dipole) in the multipole expansion. Analytical formulae and corresponding strength parameters are known for higher order terms (DDQ, dipole-dipole-quadrupole, DQQ, dipole-quadrupole-quadrupole,...) as well.

More accurate three-body potentials can be obtained using quantum chemical $a b$ initio calculations Malijevský et al. (2007). 


\section{The virial equation of state}

The virial equation of state in the statistical thermodynamics is an expansion of the compressibility factor $z=\frac{p V}{R T}$ in powers of density $\rho=\frac{N}{V}$

$$
z=1+B_{2} \rho+B_{3} \rho^{2}+\cdots,
$$

where $B_{2}$ is the second virial coefficient, $B_{3}$ the third, e.t.c. The virial coefficients of pure gases are functions of temperature only. For mixtures they are functions of temperature and composition.

The first term in equation (102) gives the equation of state of ideal gas, the first two terms or three give corrections to non-ideality. Higher virial coefficients are not available experimentally. However, they can be determined from knowledge of intermolecular forces. The relations among the intermolecular forces and the virial coefficients are exact, the pair and the three-body of potentials are subjects of uncertainties, however.

\subsection{Second virial coefficient}

For the second virial coefficient of spherically symmetric molecules we find

$$
B=-2 \pi \int_{0}^{\infty} f(r) r^{2} \mathrm{~d} r=-2 \pi \int_{0}^{\infty}\left(e^{-\beta u(r)}-1\right) r^{2} \mathrm{~d} r,
$$

where

$$
f(r)=\exp [-\beta u(r)]-1
$$

is the Mayer function. For linear molecules we have

$$
B=-\frac{1}{4} \int_{0}^{\infty} \int_{0}^{\pi} \int_{0}^{\pi} \int_{0}^{2 \pi}\left[e^{-\beta u\left(r, \theta_{1}, \theta_{2}, \phi\right)}-1\right] r^{2} \sin \theta_{1} \sin \theta_{2} \mathrm{~d} r \mathrm{~d} \theta_{1} \mathrm{~d} \theta_{2} \mathrm{~d} \phi .
$$

For general non-spherical molecules we obtain

$$
B=-\frac{2 \pi}{\int_{\vec{\omega}_{1}} \int_{\vec{\omega}_{2}} \mathrm{~d} \vec{\omega}_{1} \mathrm{~d} \vec{\omega}_{2}} \int_{0}^{\infty} \int_{\omega_{1}} \int_{\omega_{2}}\left[e^{-\beta u\left(r, \vec{\omega}_{1}, \vec{\omega}_{2}\right)}-1\right] r^{2} \mathrm{~d} r \mathrm{~d} \vec{\omega}_{1} \mathrm{~d} \vec{\omega}_{2} .
$$

\subsection{Third virial coefficient}

The third virial coefficient may be written for spherically symmetric molecules as

$$
C=C_{\text {add }}+C_{\text {nadd }},
$$

where

$$
C_{\text {add }}=-\frac{8}{3} \pi^{2} \int_{0}^{\infty} \int_{0}^{r} \int_{|r-s|}^{r+s}\left(e^{-\beta u(r)}-1\right)\left(e^{-\beta u(s)}-1\right)\left(e^{-\beta u(t)}-1\right) r s t \mathrm{~d} r \mathrm{~d} s \mathrm{~d} t,
$$

and

$$
C_{\text {nadd }}=\frac{8}{3} \pi^{2} \int_{0}^{\infty} \int_{0}^{r} \int_{|r-s|}^{r+s} e^{-\beta u(r)} e^{-\beta u(s)} e^{-\beta u(t)}\left\{\exp \left[-\beta u_{3}(r, s, t)\right]-1\right\} r s t \mathrm{~d} r \mathrm{~d} s \mathrm{~d} t,
$$

where $u_{3}(r, s, t)$ is the three-body potential. Analogous equations hold for non-spherical molecules. 


\subsection{Higher virial coefficients}

Expressions for higher virial coefficients become more and more complicated due to an increasing dimensionality of the corresponding integrals and their number. For example, the ninth virial coefficient consists of 194066 integrals with the Mayer integrands, and their dimensionalities are up to 21 Malijevský \& Kolafa (2008) in a simplest case of spherically symmetric molecules. For hard spheres the virial coefficients are known up to ten, which is at the edge of a present computer technology Labík et al. (2005).

\subsection{Virial coefficients of mixtures}

For binary mixture of components 1 and 2 the second virial coefficient reads

$$
B_{2}=x_{1}^{2} B_{2}(11)+2 x_{1} x_{2} B_{2}(12)+x_{2}^{2} B_{2}(22),
$$

where $x_{i}$ are the mole fractions, $B_{2}(i i)$ the second virial coefficients of pure components and $B_{2}(12)$ the crossed virial coefficient representing an influence of the interaction between molecule 1 and molecule 2 .

The third virial coefficient reads

$$
B_{3}=x_{1}^{3} B_{3}(111)+3 x_{1}^{2} x_{2} B_{3}(112)+3 x_{1} x_{2}^{2} B_{3}(122)+x_{2}^{3} B_{3}(222) .
$$

Extensions of these equations on multicomponent mixtures and higher virial coefficients is straightforward.

\section{Dense gas and liquid}

Determination of thermodynamic properties from intermolecular interactions is much more difficult for dense fluids (for gases at high densities and for liquids) than for rare gases and solids. This fact can be explained using a definition of the Helmholtz free energy

$$
A=U-T S .
$$

Free energy has a minimum in equilibrium at constant temperature and volume. At high temperatures and low densities the term $T S$ dominates because not only temperature but also entropy is high. A minimum in $A$ corresponds to a maximum in $S$ and system, thus, is in the gas phase. Ideal gas properties may be calculated from a behavior of individual molecules only. At somewhat higher densities thermodynamic quantities can be expanded from their ideal-gas values using the virial expansion.

At low temperatures the energy term in equation (111) dominates because not only temperature but also entropy is small. For solids we may start from a concept of the ideal crystal.

No such simple molecular model as the ideal gas or the ideal crystal is known for liquid and dense gas. Theoretical studies of liquid properties are difficult and uncompleted up to now.

\subsection{Internal structure of fluid}

There is no internal structure of molecules in the ideal gas. There is a long-range order in the crystal. The fluid is between of the two extremal cases: it has a local order at short intermolecular distances (as crystal) and a long-range disorder (as gas).

The fundamental quantity describing the internal structure of fluid is the pair distribution function $g(r)$ 


$$
g(r)=\frac{\rho(r)}{\rho},
$$

where $\rho(r)$ is local density at distance $r$ from the center of a given molecule, and $\rho$ is the average or macroscopic density of system. Here and in the next pages of this section we assume spherically symmetric interactions and the rule of the pair additivity of the intermolecular potential energy.

The pair distribution function may be written in terms of the intermolecular interaction energy $u_{N}$

$$
g(r)=V^{2} \frac{\int_{(V)} \cdots \int_{(V)} e^{-\beta u_{N}\left(\vec{r}_{1}, \vec{r}_{2}, \ldots, \vec{r}_{N}\right)} \mathrm{d} \vec{r}_{3} \ldots \mathrm{d} \vec{r}_{N}}{\int_{(V)} \cdots \int_{(V)} e^{-\beta u_{N}\left(\vec{r}_{1}, \vec{r}_{2}, \ldots, \vec{r}_{N}\right)} \mathrm{d} \vec{r}_{1} \ldots \mathrm{d} \vec{r}_{N}} .
$$

It is related to the thermodynamic quantities using the pressure equation

$$
z \equiv \frac{p V}{R T}=1-\frac{2}{3} \pi \rho \beta \int_{0}^{\infty} \frac{d u(r)}{\mathrm{d} r} g(r) r^{3} \mathrm{~d} r
$$

the energy equation

$$
\frac{U}{R T}=\frac{U^{0}}{R T}+2 \pi \rho \beta \int_{0}^{\infty} u(r) g(r) r^{2} \mathrm{~d} r,
$$

where $U^{0}$ internal energy if the ideal gas, and the compressibility equation

$$
\beta\left(\frac{\partial p}{\partial \rho}\right)_{\beta}=\left\{1+4 \pi \rho \int_{0}^{\infty}[g(r)-1] r^{2} \mathrm{~d} r\right\}^{-1} .
$$

Present mainstream theories of liquids can be divided into two large groups: perturbation theories and integral equation theories Hansen \& McDonald (2006), Martynov (1992).

\subsection{Perturbation theories}

A starting point of the perturbation theories is a separation of the intermolecular potential into two parts: a harsh, short-range repulsion and a smoothly varying long-range attraction

$$
u(r)=u^{0}(r)+u^{p}(r) .
$$

The term $u^{0}(r)$ is called the reference potential and the term $u^{p}(r)$ the perturbation potential. In the simplest case of the first order expansion of the Helmholtz free energy in the perturbation potential it holds

$$
\frac{A}{R T}=\frac{A^{0}}{R T}+2 \pi \rho \beta \int_{0}^{\infty} u^{p}(r) g^{0}(r) r^{2} \mathrm{~d} r,
$$

where $A^{0}$ is the Helmholtz free energy of a reference system.

In the perturbation theories knowledge of the pair distribution function and the Helmholtz free energy of the reference system is supposed. On one hand the reference system should be simple (the ideal gas is too simple and brings nothing new; a typical reference system is a fluid of hard spheres), and the perturbation potential should be small on the other hand. As a result of a battle between a simplicity of the reference potential (one must know its structural and thermodynamic properties) and an accuracy of a truncated expansion, a number of methods have been developed. 


\subsection{Integral equation theories}

Among the integral equation theories the most popular are those based on the Ornstein-Zernike equation

$$
h\left(r_{12}\right)=c\left(r_{12}\right)+\rho \int_{(V)} h\left(r_{13}\right) c\left(r_{32}\right) \mathrm{d} \overrightarrow{r_{3}} .
$$

where $h(r)=g(r)-1$ is the total correlation function and $c(r)$ the direct correlation function. This equation must be closed using a relation between the total and the direct correlation functions called the closure to the Ornstein-Zernike equation. From the diagrammatic analysis it follows

$$
h(r)=\exp [-\beta u(r)+\gamma(r)+B(r)]-1,
$$

where

$$
\gamma(r)=h(r)-c(r)
$$

is the indirect (chain) correlation function and $B(r)$ is the bridge function, a sum of elementary diagrams. Equation (120) does not yet provide a closure. It must be completed by an approximation for the bridge function. The mostly used closures are in listed in Malijevský \& Kolafa (2008). The simplest of them are the hypernetted chain approximation

$$
B(r)=0
$$

and the Percus-Yevick approximation

$$
B(r)=\gamma(r)-\ln [\gamma(r)+1] .
$$

Let us compare the perturbation and the integral equation theories. The first ones are simpler but they need an extra input - the structural and thermodynamic properties of a reference system. The accuracy of the second ones depends on a chosen closure. Their examples shown here, the hypernetted chain and the Percus-Yevick, are too simple to be accurate.

\subsection{Computer simulations}

Besides the above theoretical approaches there is another route to the thermodynamic quantities called the computer experiments or pseudoexperiments or simply simulations. For a given pair intermolecular potential they provide values of thermodynamic functions in the dependence on the state variables. In this sense they have characteristics of real experiments. Similarly to them they do not give an explanation of the bulk behavior of matter but they serve as tests of approximative theories. The thermodynamic values are free of approximations, or more precisely, their approximations such as a finite number of molecules in the basic box or a finite number of generated configurations can be systematically improved Kolafa et al. (2002). The computer simulations are divided into two groups: the Monte Carlo simulations and the molecular dynamic simulations. The Monte Carlo simulations generate the ensemble averages of structural and thermodynamic functions while the molecular dynamics simulations generate their time averages. The methods are described in detail in the monograph of Allen and Tildesley Allen \& Tildesley (1987).

\section{Interpretation of thermodynamic laws}

In Section 2 the axioms of the classical or phenomenological thermodynamics have been listed. The statistical thermodynamics not only determines the thermodynamic quantities from knowledge of the intermolecular forces but also allows an interpretation of the phenomenological axioms. 


\subsection{Axiom on existence of the thermodynamic equilibrium}

This axiom can be explained as follows. There is a very, very large number of microscopic states that correspond to a given macroscopic state. At unchained macroscopic parameters such as volume and temperature of a closed system there is much more equilibrium states then the states out of equilibrium. Consequently, a spontaneous transfer from non-equilibrium to equilibrium has a very, very high probability. However, a spontaneous transfer from an equilibrium state to a non-equilibrium state is not excluded.

Imagine a glass of whisky on rocks. This two-phase system at a room temperature transfers spontaneously to the one-phase system - a solution of water, ethanol and other components. It is not excluded but it is highly improbable that a glass with a dissolved ice will return to the initial state.

\subsection{Axiom of additivity}

This axiom postulates that the internal energy of the macroscopic system is a sum of its two macroscopic parts

$$
U=U_{1}+U_{2} \text {. }
$$

However, if we consider a macroscopic system as an assembly of molecules, equation (123) does not take into account intermolecular interactions among molecules of subsystem 1 and molecules of subsystem 2 . Correctly the equation should be

$$
U=U_{1}+U_{2}+U_{12} .
$$

Due to the fact that intermolecular interactions vanish at distances of the order of a few molecule diameters, the term $U_{12}$ is negligible in comparison with $U$.

\subsection{The zeroth law of thermodynamics and the negative absolute temperatures}

The statistical thermodynamics introduces temperature formally as parameter $\beta=\frac{1}{k_{B} T}$ in the expression (11) for the partition function

$$
Q=\sum_{i} \exp \left(-\beta E_{i}\right)
$$

As energies of molecular systems are positive and unbounded, temperature must be positive otherwise the equation diverges. For systems with bounded energies

$$
E_{\min } \leq E_{i} \leq E_{\max }
$$

both negative and positive temperatures are allowed. Such systems are in lasers, for example.

\subsection{The second law of thermodynamics}

From equation (3) it follows that entropy of the adiabatically isolated system either grows for spontaneous processes or remains constant in equilibrium

$$
\mathrm{d} S \geq 0 \text {. }
$$

Entropy in the statistical thermodynamics is connected with probability via equation (20)

$$
S=-k_{B} \sum_{i} P_{i} \ln P_{i} .
$$

Thus, entropy may spontaneously decrease but with a low probability. 


\subsection{Statistical thermodynamics and the arrow of time}

Direction of time from past to future is supported by three arguments

- Cosmological time

The cosmological time goes according the standard model of Universe from the Big Bang to future.

- Psychological time

We as human beings remember (as a rule) what was yesterday but we do not "remember" what will be tomorrow.

- Thermodynamic time

Time goes in the direction of the growth of entropy in the direction given by equation (125). The statistical thermodynamics allows due to its probabilistic nature a change of a direction of time "from coffin to the cradle" but again with a very, very low probability.

\subsection{The third law of thermodynamics}

Within the statistical thermodynamics the third law may be easily derived from equation (20) relating entropy and the probabilities. The state of the ideal crystal at $T=0 \mathrm{~K}$ is one. Its probability $P_{0}=1$. By substituting to the equation we get $S=0$.

\section{Acknowledgement}

This work was supported by the Ministry of Education, Youth and Sports of the Czech Republic under the project No. 6046137316.

List of symbols

A Helmholtz free energy

B second virial coefficient

$\mathrm{B}_{i} \quad$ i-th virial coefficient

$B(r)$ bridge function

$\mathrm{C}_{V}$ isochoric heat capacity

$\mathrm{C}_{p}$ isobaric capacity

$c(r)$ direct correlation function

E energy

$\epsilon \quad$ energy of molecule

G Gibbs free energy

$g(r)$ pair distribution function

$\mathrm{H}$ enthalpy

h Planck constant

$h(r)$ total correlation function

$\mathrm{k}_{B} \quad$ Boltzmann constant

$\mathrm{N}$ number of molecules, Avogadro number

$\mathrm{P}$ probability

$\mathrm{p}$ pressure

Q heat

Q partition function

q partition function of molecule

$\mathrm{R}$ (universal) gas constant (8.314 in SI units)

S entropy

$\mathrm{T}$ temperature 


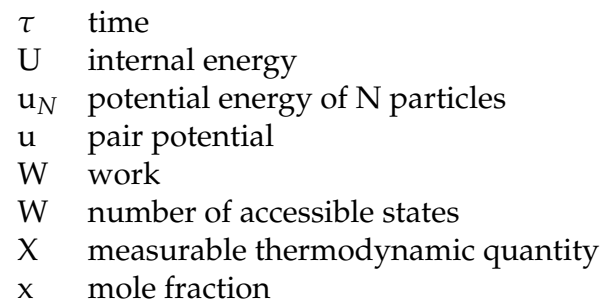

\section{References}

Feynman, R. P.; Leghton, R. B. \& Sands, M. (2006). The Feynman lectures on physics,Vol. 1, Pearson, ISBN 0-8053-9046-4, San Francisco.

Lucas K. (1991). Applied Statistical Thermodynamics, Springer-Verlag, ISBN 0-387-52007-4, New York, Berlin, Heidelberg.

Aziz, R.A. (1984). Interatomic Potentials for Rare-Gases: Pure and Mixed Interactions, In: Inert Gases M. L. Klein (Ed.), 5 - 86, Springer-Verlag, ISBN 3-540-13128-0, Berlin, Heidelberg.

Slaviček, P.; Kalus, R.; Paška, P.; Odvárková, I.; Hobza, P. \& Malijevský, A. (2003). State-of-the-art correlated ab initio potential energy curves for heavy rare gas dimers:Ar, $\mathrm{Kr}_{2}$, and $\mathrm{Xe}_{2}$. Journal of Chemical Physics, 119, 4, 2102-2119, ISSN:0021-9606.

Malijevský, Alexandr; Karlický, F.; Kalus, R. \& Malijevský, A. (2007). Third Viral Coeffcients of Argon from First Principles. Journal of Physical Chemistry C, 111, 43, 15565 - 15568, ISSN:1932-7447.

Malijevský, A. \& Kolafa, J. (2008). Introduction to the thermodynamics of Hard Spheres and Related Systems In: Theory and Simulation of Hard-Sphere Fluids and Related Systems A. Mulero (Ed.) 27 - 36, Springer-Verlag, ISBN 978-3-540-78766-2, Berlin, Heidelberg.

Labík, S.; Kolafa, J. \& Malijevský, A. (2005). Virial coefficients of hard spheres and hard disks up to the ninth. Physical Review E, 71, 2-1, 021105/1-021105/8, ISSN:1539-3755.

Allen, M. P. \& Tildesley, D. J. (1987). Computer Simulation of Liquids, Claredon Press, ISBN: 0-19-855645-4, Oxford.

Hansen, J.-P. \& McDonald, I. R. (2006). Theory of Simple Fluids, Elsevier, ISBN: 978-0-12-370535-8, Amsterdam.

Martynov, G. A. (1992). Fundamental Theory of Liquids, Adam Hilger, ISBN: 0-7503-0069-8, Bristol, Philadelphia and New York.

Malijevský, A. \& Kolafa, J. (2008). Structure of Hard Spheres and Related Systems In: Theory and Simulation of Hard-Sphere Fluids and Related Systems A. Mulero (Ed.) 1 - 26, Springer-Verlag, ISBN 978-3-540-78766-2, Berlin, Heidelberg.

Kolafa, J.; Labík \& Malijevský, A. (2002). The bridge function of hard spheres by direct inversion of computer simulation data. Molecular Physics, 100, 16, 2629 - 2640, ISSN: 0026-8976.

Baus, M.\& Tejero, C., F. (2008). Equilibrium Statistical Physics: Phases of Matter and Phase Transitions, Springer, ISBN: 978-3-540-74631-7, Heidelberg.

Ben-Naim, A. (2010). Statistical Thermodynamics Based on Information: A Farewell to Entropy, World-Scientific, ISBN: 978-981-270-707-9, Philadelphia, New York.

Plischke, M. \& Bergsen, B. (2006). Equilibrium Statistical Physics, University of British Columbia, Canada, ISBN: 978-981-256-048-3. 


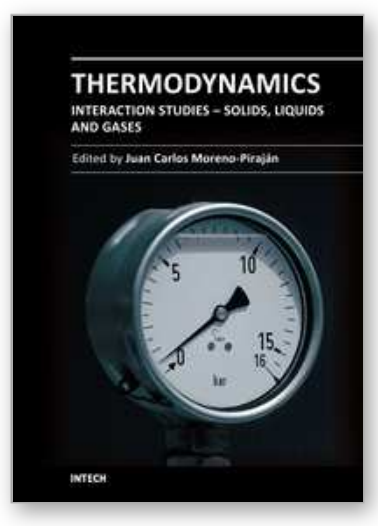

\author{
Thermodynamics - Interaction Studies - Solids, Liquids and Gases \\ Edited by Dr. Juan Carlos Moreno PirajÃ $\tilde{A}_{j}$
}

ISBN 978-953-307-563-1

Hard cover, 918 pages

Publisher InTech

Published online 02, November, 2011

Published in print edition November, 2011

Thermodynamics is one of the most exciting branches of physical chemistry which has greatly contributed to the modern science. Being concentrated on a wide range of applications of thermodynamics, this book gathers a series of contributions by the finest scientists in the world, gathered in an orderly manner. It can be used in post-graduate courses for students and as a reference book, as it is written in a language pleasing to the reader. It can also serve as a reference material for researchers to whom the thermodynamics is one of the area of interest.

\title{
How to reference
}

In order to correctly reference this scholarly work, feel free to copy and paste the following:

Anatol Malijevsky (2011). Statistical Thermodynamics, Thermodynamics - Interaction Studies - Solids, Liquids and Gases, Dr. Juan Carlos Moreno PirajÃ $i n$ (Ed.), ISBN: 978-953-307-563-1, InTech, Available from:

http://www.intechopen.com/books/thermodynamics-interaction-studies-solids-liquids-and-gases/statisticalthermodynamics

\section{INTECH}

open science | open minds

\section{InTech Europe}

University Campus STeP Ri

Slavka Krautzeka 83/A

51000 Rijeka, Croatia

Phone: +385 (51) 770447

Fax: +385 (51) 686166

www.intechopen.com

\section{InTech China}

Unit 405, Office Block, Hotel Equatorial Shanghai

No.65, Yan An Road (West), Shanghai, 200040, China

中国上海市延安西路65号上海国际贵都大饭店办公楼 405 单元

Phone: +86-21-62489820

Fax: +86-21-62489821 
(C) 2011 The Author(s). Licensee IntechOpen. This is an open access article distributed under the terms of the Creative Commons Attribution 3.0 License, which permits unrestricted use, distribution, and reproduction in any medium, provided the original work is properly cited. 